\title{
Platinum chemotherapy for BRCA1-related breast cancer: do we need more evidence?
}

\author{
Nicholas C Turner ${ }^{* 1,2}$ and Andrew NJ Tutt ${ }^{3}$ \\ See related research by Byrski et al., http://breast-cancer-research.com/content/14/4/R110
}

\begin{abstract}
A recent prospective clinical trial provides further evidence that breast cancers arising in germline BRCA1 mutation carriers are highly sensitive to cisplatin chemotherapy. The potential significance of these data for the management of patients with BRCA1-related and BRCA2-related breast cancer is discussed.
\end{abstract}

In a previous issue of Breast Cancer Research Tomasz Byrski and colleagues present the results of a prospective phase II study of cisplatin in BRCA1-related metastatic breast cancer - that is, breast cancer arising in women with a germline mutation in BRCA1 [1]. They report evidence of substantial efficacy with an overall response rate of $80 \%$, including $45 \%$ with complete response, and a time to progression of 12 months. The majority of patients in the study had triple receptor-negative breast cancer, and this time to progression compares favorably with median progression-free survival for triple receptornegative breast cancer in contemporary series [2]. This study follows on from a retrospective study by the same group that reported a pathological complete response rate of $83 \%$ with neoadjuvant cisplatin chemotherapy, compared with a rate of $15 \%$ with nonrandomized comparator neoadjuvant chemotherapy [3].

The molecular basis for these high response rates is well understood. Both BRCA1 and BRCA2 are required for DNA double-strand break repair by homologous recombination (HR-based DNA repair) [4,5]. Mutations in BRCA1 and BRCA2 inactivate protein function, and in cancer the wild-type allele is almost invariably lost, leading to a defect in HR-based DNA repair in the cancer. Platinum chemotherapy generates interstrand cross-links

*Correspondence: nicholas.turner@icr.ac.uk

'The Breakthrough Breast Cancer Research Centre, Institute of Cancer Research,

237 Fulham Road, London SW3 6JB, UK

Full list of author information is available at the end of the article that can only be adequately repaired by HR-based DNA repair, and consequently BRCA1-deficient and BRCA2deficient cells are highly sensitive to platinum chemotherapy both in vitro and in vivo. With high response rates in a prospective clinical trial, and a strong biological rationale, it is time to ask whether we are moving towards a new chemotherapy standard for $B R C A 1$-related, and potentially by inference BRCA2related, breast cancer or whether we need more evidence.

The main strength of the current study is that it has been carried out at all. BRCA1 mutations account for a small proportion of patients with advanced breast cancer, even in countries with founder mutations, and this presents a substantial barrier to running studies testing standard chemotherapy. Use of the chemotherapy regimen outside the trial, and the wide availability of novel therapy trials competing for the same patients, add to the challenges of recruiting such trials. Nevertheless, the study by Byrski and colleagues is an open-label singlearm study of only 20 patients, with no central radiological confirmation of response rates, and both this study design and this size make a meaningful interpretation of progression-free survival very difficult. The study was in addition not prospectively registered in a clinical trial registry, removing one of the safeguards against publication bias.

The study is dominated by women with three specific mutations in BRCA1 that represent the three founder mutations found in the Polish population [1], with over one-half being the single mutation 5382insC. One of these mutations, C61G, is predicted not to sensitize to cisplatin on the basis of preclinical data [6] yet cancers with this mutation appear to be just as sensitive to cisplatin in the study [1], a discrepancy for which it is important to understand the basis. Prior studies reported by this group have also been drawn from the Polish founder mutations, and we have limited data on the response of cancers with other BRCA1 mutations, and very limited data for $B R C A 2$ mutations.

Although the data for $B R C A 1 / 2$-related breast are therefore relatively limited, there are substantial data on the sensitivity of BRCA1-related and BRCA2-related 
ovarian cancers to platinum-based chemotherapy $[7,8]$. $B R C A 1 / 2$-related serous ovarian cancers are highly sensitive to platinum chemotherapy, and remain sensitive to repeat challenges with platinum chemotherapy, which likely explains the improved survival of $B R C A 1 / 2$-related serous ovarian cancer compared with $B R C A 1 / 2$ wildtype serous ovarian cancer [8].

Should the accumulation of data, which includes this study by Byrski and colleagues, alter our approach to the treatment of BRCA1-related and BRCA2-related breast cancer? For patients with metastatic BRCA1-related breast cancer, although the data are limited, it seems clear that these patients should be offered the option of platinum-containing chemotherapy at some point during their treatment course. Whether platinum chemotherapy should be used as the first line in preference to other chemotherapy agents is unclear, and this is the subject of the BRCA trial (NCT00321633, NCT00532727) that randomizes first-line patients between carboplatin and docetaxel. For those with BRCA1 mutation-associated triple receptor-negative breast cancer and anthracyclineresistant and taxane-resistant disease, where there are few available active therapies, and the option of platinumagent chemotherapy seems well founded.

Whether the platinum agent should be cisplatin or whether carboplatin would have a similar response rate is unknown. Any difference in efficacy between the two drugs is likely to be small and may be outweighed by logistical and toxicity advantages for the patient. Whether patients with evidence of disease response and a long platinum-free interval ( $>6$ months off chemotherapy) should be retreated with platinum-based chemotherapy on progression or whether they should be treated with alternative chemotherapy regimens remains unclear, and we await data to guide such decisions. Although there are few direct data on BRCA2-related breast cancer, the strength of the biological rational, the comparative data between $B R C A 1$ and $B R C A 2$ in ovarian cancer, and the evidence of poly(ADP ribose) polymerase (PARP) inhibitor efficacy in $B R C A 2$-related breast cancer [9] all suggest that similar advice should apply to BRCA2related breast cancer.

What about the curative setting and patients receiving adjuvant or neoadjuvant chemotherapy? Here the data are less robust. Standard adjuvant anthracycline/taxane chemotherapy cures a substantial proportion of women with breast cancer, with evidence of better outcomes and therapy responses in the $B R C A 1 / 2$ carrier population $[10,11]$, so changes to this standard should only be made on the basis of strong evidence. At present the data to support platinum agents in this context are limited to retrospective analysis [3] or to prospective data for a very small number of patients [12]. Prospective studies are still required before routine practice changes in the curative setting. The one current exception to this is in the treatment of HER2-positive breast cancer in BRCA1/2 carriers. Relative equipoise has already been shown in the general breast cancer population for the TCH (docetaxel, carboplatin, trastuzumab) regimen compared with standard anthracycline-taxane-trastuzumab-based chemotherapy [13], and the TCH (docetaxel, carboplatin, trastuzumab) regimen presents an attractive option for $B R C A 1 / 2$ carriers with HER2-positive breast cancer.

PARP inhibitors target the same HR-based DNA repair defect as cisplatin chemotherapy, and there is evidence of efficacy for the PARP inhibitor olaparib in BRCA1-related and $B R C A 2$-related breast cancer with substantial prior chemotherapy exposure [9]. PARP inhibitors target the DNA repair defect in a more specific fashion and are well tolerated without typical chemotherapy side effects [9]. The challenge in BRCA1/2-related advanced breast cancer is to develop and support a collaborative mechanism where patients can be identified and entered into randomized trials that test novel therapies such as PARP inhibitors, or mechanistically based chemotherapy, to robustly assess the efficacy relative to standard care, and therefore allow these patients to benefit from these $B R C A 1 / 2$-focused treatments.

Abbreviations

$H R$, homologous recombination; PARP, poly(ADP ribose) polymerase.

Competing interests

The authors declare that they have no competing interests.

\section{Authors' details}

'The Breakthrough Breast Cancer Research Centre, Institute of Cancer Research, 237 Fulham Road, London SW3 6JB, UK. 'Breast Unit, Royal Marsden Hospital, Fulham Road, London SW3 6JJ, UK. ${ }^{3}$ Breakthrough Breast Cancer Research Unit, Guy's Hospital Campus, Kings Health Partners, London SE1 9RT, UK.

Published: 13 November 2012

\section{References}

1. Byrski T, Dent R, Blecharz P, Foszczynska-Kloda M, Gronwald J, Huzarski T, Cybulski C, Marczyk E, Chrzan R, Eisen A, Lubinski J, Narod SA: Results of a phase II open-label, non-randomized trial of cisplatin chemotherapy in patients with BRCA1-positive metastatic breast cancer. Breast Cancer Res 2012, 14:R110

2. O'Shaughnessy J, Osborne C, Pippen JE, Yoffe M, Patt D, Rocha C, Koo IC Sherman BM, Bradley C: Iniparib plus chemotherapy in metastatic triplenegative breast cancer. N Engl J Med 2011, 364:205-214.

3. Byrski T, Gronwald J, Huzarski T, Grzybowska E, Budryk M, Stawicka M, Mierzwa T, Szwiec M, Wisniowski R, Siolek M, Dent R, Lubinski J, Narod S: Pathologic complete response rates in young women with BRCA1positive breast cancers after neoadjuvant chemotherapy. J Clin Oncol 2010, 28:375-379.

4. Moynahan ME, Chiu JW, Koller BH, Jasin M: Brca1 controls homologydirected DNA repair. Mol Cell 1999, 4:511-518.

5. Tutt A, Bertwistle D, Valentine J, Gabriel A, Swift S, Ross G, Griffin C, Thacker J, Ashworth A: Mutation in Brca2 stimulates error-prone homology-directed repair of DNA double-strand breaks occurring between repeated sequences. EMBO J 2001, 20:4704-4716.

6. Drost R, Bouwman P, Rottenberg S, Boon U, Schut E, Klarenbeek S, Klijn C, van der Heijden I, van der Gulden H, Wientjens E, Pieterse M, Catteau A, Green P, Solomon E, Morris JR, Jonkers J: BRCA1 RING function is essential for tumor 
suppression but dispensable for therapy resistance. Cancer Cell 2011, 20:797-809.

7. Alsop K, Fereday S, Meldrum C, Defazio A, Emmanuel C, George J, Dobrovic A, Birrer MJ, Webb PM, Stewart C, Friedlander M, Fox S, Bowtell D, Mitchell G: BRCA mutation frequency and patterns of treatment response in BRCA mutation-positive women with ovarian cancer: a report from the Australian Ovarian Cancer Study Group. J Clin Oncol 2012, 30:2654-2663.

8. Tan DS, Rothermundt C, Thomas K, Bancroft E, Eeles R, Shanley S, ArdernJones A, Norman A, Kaye SB, Gore ME: 'BRCAness' syndrome in ovarian cancer: a case-control study describing the clinical features and outcome of patients with epithelial ovarian cancer associated with BRCA1 and BRCA2 mutations. J Clin Oncol 2008, 26:5530-5536.

9. Tutt A, Robson M, Garber JE, Domchek SM, Audeh MW, Weitzel JN, Friedlander M, Arun B, Loman N, Schmutzler RK, Wardley A, Mitchell G, Earl H, Wickens M, Carmichael J: Oral poly(ADP-ribose) polymerase inhibitor olaparib in patients with BRCA1 or BRCA2 mutations and advanced breast cancer: a proof-of-concept trial. Lancet 2010, 376:235-244.

10. Arun B, Bayraktar S, Liu DD, Gutierrez Barrera AM, Atchley D, Pusztai L, Litton JK, Valero V, Meric-Bernstam F, Hortobagyi GN, Albarracin C: Response to neoadjuvant systemic therapy for breast cancer in BRCA mutation carriers and noncarriers: a single-institution experience. J Clin Onco/ 2011, 29:3739-3746
11. Gonzalez-Angulo AM, Timms KM, Liu S, Chen H, Litton JK, Potter J, Lanchbury JS, Stemke-Hale K, Hennessy BT, Arun BK, Hortobagyi GN, Do KA, Mills GB, Meric-Bernstam F: Incidence and outcome of BRCA mutations in unselected patients with triple receptor-negative breast cancer. Clin Cancer Res 2011, 17:1082-1089.

12. Silver DP, Richardson AL, Eklund AC, Wang ZC, Szallasi Z, Li Q, Juul N, Leong CO, Calogrias D, Buraimoh A, Fatima A, Gelman RS, Ryan PD, Tung NM, De Nicolo A, Ganesan S, Miron A, Colin C, Sgroi DC, Ellisen LW, Winer EP, Garber JE: Efficacy of neoadjuvant cisplatin in triple-negative breast cancer. J Clin Oncol 2010, 28:1145-1153

13. Slamon D, Eiermann W, Robert N, Pienkowski T, Martin M, Press M, Mackey J, Glaspy J, Chan A, Pawlicki M, Pinter T, Valero V, Liu MC, Sauter G, von Minckwitz G, Visco F, Bee V, Buyse M, Bendahmane B, Tabah-Fisch I, Lindsay MA, Riva A, Crown J: Adjuvant trastuzumab in HER2-positive breast cancer. NEngl J Med 2011, 365:1273-1283.

doi:10.1186/bcr3332

Cite this article as: Turner NC, Tutt ANJ.: Platinum chemotherapy for BRCA1related breast cancer: do we need more evidence? Breast Cancer Research 2012, 14:115 